\title{
Percepción de los Estudiantes de Ingeniería, Tecnología y Curso Técnico sobre Responsabilidad Social Empresarial
}

\section{Perception of Students in Engineering, Technology and Technical Course on Corporate Social Responsibility}

\author{
Rosângela de Fátima Stankowitz Penteado', Leila Mendes da Luz², Patrício Henrique de Vasconcelos ${ }^{3}$, \\ Hélio Gomes de Carvalho ${ }^{4}$,Antonio Carlos de Francisco ${ }^{5}$
}

\begin{abstract}
Este artículo identifica la percepción de los alumnos de Ingeniería, Tecnología y Técnico de la Universidad Tecnológica Federal del Paraná (UTFPR) sobre la Responsabilidad Social (RS) mediante un cuestionario estructurado en tres ejes: i) Percepción sobre RS en las empresas; ii) En relación a UTFPR e, iii) RS sobre sí mismos. Por un lado, las acciones deben ser compartidas con acciones gubernamentales en la promoción del bien estar y desarrollo social, por ende, las empresas deben de responsabilizarse y resolver los problemas sociales. Por otro lado, las acciones y proyectos de la institución son percibidos para el posicionamiento Social responsable en la formación profesional. Existe una RS en la calidad de compras y las mejores prácticas son las que preservan el medio ambiente, el reciclaje y respetan a los derechos humanos.
\end{abstract}

Keywords: responsabilidad social, universidad, empresas.

This article identifies the perceptions of students in Engineering, Technology and Technical Course at the Federal University of Technology, Paraná (UTFPR), Campus Ponta Grossa, on Social Responsibility (SR) using a structured questionnaire in three areas: i) perception of CSR in companies, ii) in relation to UTFPR, and iii) RS about themselves. Actions should be shared with the government's actions in promoting social welfare and development. Companies should be accountable and solve their social problems. Actions and projects of the institution are perceived with a positioning responsible socially in the formation. Exist a RS in shopping quality and the best practices are those that preserve the environment, recycle and respect human rights.

Keywords: social responsibility, university, companies.

Departamento de Ingeniería de Producción, Universidad Tecnológica Federal del Paraná, Av. Monteiro Lobato, s/n - Km04, Ponta Grossa, Paraná, Brasil, CEP: 840I6-210, Phone: +55 (42) 3220-4876. Email: 'rstankowitzp@gmail.com, 2leila.mendesdaluz@gmail.com, 3adm.patricio@yahoo.com.br, ${ }^{3}$ helio@utfpr.edu.br, ${ }^{5}$ acfrancisco@utfpr.edu.br 


\section{Introducción}

El mundo está pasando por procesos de cambios rápidos. Las relaciones económicas, las innovaciones tecnológicas y los valores de la sociedad reflecten en la estructura y el ambiente organizacional.

Grayson (2002), defiende que las fuerzas globales de estos cambios surgieron con la revolución de la tecnológica, de los mercados, de la revolución de la demografía y del desarrollo, principalmente, con la revolución de los valores. Esta última, basada en ideas y percepciones provocó cambios en la naturaleza de los negocios, en el comportamiento de las personas y del papel del Estado.

Cada vez más, publicaciones en revistas especializadas, las investigaciones académicas y los medios de comunicación en general, revelan la tendencia creciente del ejercicio de la Responsabilidad Social Empresarial (RSE) por parte de las organizaciones y la ansiedad por parte de la Sociedad para que las organizaciones cumplan los principios de la RSE.

Para Tachizawa (2008), la expansión de la consciencia colectiva con relación a las cuestiones ambientales y las demandas sociales inducirán un nuevo posicionamiento de parte de empresarios y ejecutivos contra estas cuestiones, creando relaciones más próximas entre las empresas, los consumidores y las comunidades en que estas actúan más directamente.

De esta forma, cuando se habla en RSE, debemos de entenderla bajo la óptica de varias dimensiones e ideas; para algunos, está relacionada al cumplimento de la ley, para otros, con las acciones sociales de las empresas filantrópicas o con la ética empresarial. En efecto, todos estos elementos citados, componen la Responsabilidad Social Empresarial, desde que no sea un fin en sí mismo. Estas son partes integrantes de una visión mayor que entiende la RS, como una forma de gestión definida por la relación ética y transparente de la empresa con los públicos con que está relacionada. En este concepto está incluido el establecimiento de metas que estimulen el desarrollo sustentable de la sociedad, llevando en consideración la preservación de los recursos ambientales y culturales, al mismo tiempo, respectando la diversidad en que promueve la reducción de las desigualdades sociales (Ethos, 20I I).

Por tanto, hay un abordaje conceptual complejo, dinámico, basado en valores y percepciones, por ende, comprender la RSE bajo esta óptica, depende del grado de conocimiento, información y percepción que los sujetos de la sociedad entienden sobre este tema, que muchas veces puede generar conflictos o disfunciones en cuanto al campo conceptual y práctico.
Así, las instituciones de enseñanza asumen un papel importante en la formación de los profesionales que presentan una visión más amplia, tanto como influencian en comportamientos socialmente responsables, contribuyendo de esta forma para atender las exigencias de la sociedad actual.

Delante de ese contexto, este artigo tiene como objetivo identificar cual es la percepción que los alumnos de los cursos de Ingeniería,Tecnología y Técnico de la Universidad Tecnológica Federal del Paraná, tienen acerca del tema RSE y como se comportan bajo esta óptica.

Por tanto, fueron trazados los siguientes objetivos específicos: a) Presentar un perfil socio-cultural-demográfico de los alumnos de los cursos del área tecnológica de la UTFPR campus Ponta Grossa; b) Identificar las percepciones de estos, acerca de la responsabilidad social de las empresas; c) Verificar si la formación ofrecida en los cursos de Ingeniería de la UTFPR permite la construcción de una consciencia profesional socialmente responsable.

Con eso, el presente artículo visa contribuir con la maduración del debate sobre la RSE con la medida que realiza un estudio junto a los académicos de cursos de la UTFPR, como una forma de observar hasta qué punto los futuros graduados de la institución perciben la importancia del compromiso social responsable de las empresas en razón de la nueva realidad social.

\section{Base teórica}

El tema Responsabilidad Social Empresarial (RSE) puede ser discutido en varias esferas de la percepción, sean estas éticas, legales, ambientales, sociales y/o económicas.

Sin embargo, estas percepciones tienen relación directa con el modo como cada sujeto involucrado en esta temática, este entiénde el significado de la RS y se comporta en relación a esta, siendo necesario el conocimiento conceptual para responder cuestiones como: ¿Qué viene a ser la RS? ¿Cómo interfiere en los sistemas productivos y el proceso de consumo? ¿Cómo son los futuros profesionales formados por las instituciones de enseñanza se posicionan sobre esta óptica? ¿Cuáles son los límites y las interfaces sobre esta temática?

Así, en las próximas secciones serán discutidos los abordajes sobre la RSE que puedan dar luz a las cuestiones que se presentan y que son fundamentales en el campo de las percepciones sobre este tema. 


\section{Definiciones sobre la RSE y sus interfaces}

La dinámica de la actividad empresarial unidas a la complejidad de los impactos provocados junto con el público de interés de las organizaciones, hoy denominados stakeholders, que empezaron con las primeras discusiones sobre el tema RSE. Las grandes transformaciones globales bajo los aspectos económicos, políticos y sociales influenciaron en el comportamiento de las empresas y la sociedad delante de cuestiones relacionadas a la ética empresarial, gestión social, utilización de recursos y consumo y nuevas formas de gestionar las empresas.

El concepto teórico sobre la RSE asociase a las diferentes ideas y percepciones. Los primeros debates han surgido alrededor de la década de 60 y persistió en diferentes corrientes hasta los días actuales, aunque no se tenga una definición consensual sobre la RSE es posible por medio de los modelos presentados en la literatura trazar las principales vertientes conceptuales sobre este tema.

Carrol (1991), uno de los mayores exponentes de la literatura en RSE, reunió, comparó y analizó los abordajes de los estudios académicos y sus contribuciones entre los años 1950 hasta mediados de los 1990. Los resultados apuntaron a Howard R. Bower, autor del libro "Las responsabilidades Sociales del Hombre de Negocios", como el precursor de las discusiones sobre la RSE.

Para Bower (1953), la Responsabilidad Social es la, "obligación del empresario en adoptar prácticas, tomar decisiones y acompañar las líneas de decisiones y las líneas de acción deseables según los objetivos y valores de la sociedad".

En la misma línea de pensamiento, autores como Davis, Eells eWalton son destacados por Carrol (1991) por referirse a la RSE, siendo las decisiones y acciones de interés económico o técnico tomadas por empresas con base en principios éticos para conducir las relaciones empresariales y sociales. En la visión del autor, las empresas deben establecer y practicar metas y programas sociales, integrados a la sensibilidad ética en la toma de decisiones, políticas y acciones adicionadas de cantidad, calidad, eficacia y eficiencia; resalta todavía, que en sus estudios identificaron la existencia de una creciente atención dada por los académicos y por los gestores de negocios sobre, como las empresas responderían y actuarían en relación a la RS, no apenas en el campo de los abordajes, sino en el sentido de cómo colocarlas en práctica.

Carrol (1979), expone que la Responsabilidad Social de una empresa engloba las expectativas económicas, legales, éticas y filantrópicas que una sociedad tiene de las organizaciones en un momento determinado.
Ya para el instituto Ethos (20II), institución de referencia de este tema en Brasil. La RSE, es la forma de gestión que se define por la relación ética y transparente de la empresa con todos los públicos con los cuales ella se relaciona, y por el establecimiento de metas empresariales que impulsen el desarrollo sustentable de la sociedad, preservando recursos ambientales y culturales para las generaciones futuras, respectando la diversidad y promoviendo la reducción de las desigualdades sociales. Esta visión es pautada en la relación entre la empresa y las comunidades con las cuales mantienen contacto, además, del nivel de impacto que sus acciones pueden tener en términos de actitud, aceptación o rechazo de su público interesado.

El confronto entre estas diferentes percepciones, valores y prácticas, contribuyó para la evolución del concepto y del entendimiento de lo que viene a ser RSE. Hasta mediados de 1980 las empresas adoptaban una visión económica sobre la RSE, para ellas, la única RS de la empresa y de sus ejecutivos era maximizar el lucro para los inversionistas, por lo que la preocupación central era atender a los intereses de sus dueños.

Esa visión está basada en el economista Friedman (1970), que defendió la idea que: "La única RSE era generar lucro para sus inversionistas, dentro de las reglas de la sociedad (leyes)". Se apunta también, que los papeles de la empresa y del estado en la sociedad deberían estar claramente definidos con vistas a no provocar la existencia de desvío de conducta con la finalidad de ambos. Por un lado, esa estrategia centrada en el inversionista promovió el crecimiento de la producción en masa, padrón de vida y nivel educacional, por otro, resultó también en la exploración del trabajo y la naturaleza. Son aspectos de acuerdo con los fundamentos de la RSE y contradictorios con los intereses públicos de la sociedad.

Así, delante de esa falta de consenso sobre los conceptos de la RSE, es posible entender porque un grande número de personas confunden RSE con Obligaciones Sociales, Filantropía, o todavía con Acciones Sociales, para eso, la próxima sección aborda las literaturas que tratan del tema en relación a la RSE.

\section{RSE y las Obligaciones Sociales}

La definición de Responsabilidad Social Corporativa como obligación social, tiene como su defensor al economista Milton Freedman (1970), esta definición fue objetivo de refutaciones generando críticas desfavorables. Uno de los argumentos desfavorables parte de la concepción de que el contrato social que constituye la base sobre la cual se construyó el sistema de la libre empresa cambió por causa de las demandas sociales y que las responsabilidades de una 
empresa están mucho más allá de los cumplimientos legales. Además, Friedman (1970), argumenta que las demandas sociales son de responsabilidad del Estado, que es una unidad compuesta de misión y competencia principal, promoviendo el bienestar de la sociedad.

Por esta definición, entiéndase que la RSE radica en el cumplimiento de las regulaciones y legislaciones vigentes, pago de impuestos y cumplimento de los deberes para con sus empleados; este presupuesto provocó otra discusión: ¿Porqué una empresa que cumple sus obligaciones sociales no sería considerada socialmente responsable? Para responder esta cuestión, se puede usar el concepto de Carrol (I99I), cuando dice que la Responsabilidad Social Corporativa no se limita a los aspectos legales de la conducta empresarial, sino a las diferentes relaciones que mantiene con su grupo de stakeholders (públicos de interés) y principalmente, en apoyo del Estado para el desarrollo social, que no se limita apenas en la generación de empleos, pago de impuestos, contribuciones y tasas.

Así, de acuerdo con Oliveira (2008), las obligaciones sociales tienen relación directa con las leyes laborales, tributación y contribuciones, no siendo pasible de confusión en cuanto a los términos como sinónimos de RS, por lo tanto, una organización ilegal no es considerada socialmente responsable, aunque apenas cumpla con los aspectos legales, esta no podría ser considerada socialmente responsable solo por eso. Sin embargo, parte de la sociedad, gestores y empleados de diferentes áreas corroboran con el economista Milton Freedman (1970), cuando entienden que la RS es apenas el cumplimento de los aspectos legales de un negocio.

\section{RSE, Acciones Sociales y Filantropía}

Existe una percepción sobre RSE como acciones sociales, donaciones (filantropía) y proyectos de RS desarrollados por las empresas que benefician algunos grupos, tales como comunidades, empleados y dependientes, escuelas y organizaciones no gubernamentales (ONGs).

En Brasil, según Alonso, López e Castrucci (2006), a partir de la década de los 80 , aparecieron indicios de RS en forma de acciones filantrópicas y eventuales dentro de las entidades, incluyendo el planeamiento estratégico de las empresas auxilio financiero para proyectos sociales y hasta la formación de fundaciones centradas para las necesidades de la sociedad.

Estas acciones pueden ser introducidas de manera coordinada y planeada, siendo considerada como una inversión social privada el emprendedorísmo social, cuando las organizaciones buscan traer la práctica de la acción social y principios aplicados en la gestión empresarial tradicional.
Para Oliveira (2008), a pesar que las acciones sociales tienen importancia, no puede ser el único parámetro para evaluar a la RSE, algunos emprendedoristas invisten en acciones sociales, pero como una forma de enmascarar otros problemas de orden ambiental, ético y hasta judicial; todavía es preciso tener clareza que la Filantropía preconiza una "Acción Social", pero con proyectos no ligados directamente a los negocios de la empresa, que muchas veces están ligados a las causas cuyos valores son compartidos por la empresa, gestores o dueños.

De esa forma, la filantropía es considerada la raíz de la RS pero, no se confunde con la misma por no estar inserida directamente en el planeamiento estratégico de la empresa, y principalmente, por no ser capaz de promover transformación social.

\section{RSE, Ética Social y Ética Empresarial}

La ética social estudia el comportamiento ético de los individuos, en las sociedades formadas por ellos. Ella no modifica la ética personal, sin embargo lleva en consideración que la persona vive en sociedad, vive con y entre otras personas. En último análisis, la ética es siempre personal, además de alcanzar una dimensión social, principalmente mediante los actos humanos externos que implican relación con otros.

La ética social es el conjunto de principios y valores individuales que gobiernan las sociedades (Alonso, López y Castrucci, 2006), así que, de acuerdo con la teoría ética, lo que es bueno para la persona es bueno para la sociedad. La ética puede ser confundida con las leyes, estas generalmente creadas con base en los principios éticos, caso no sean cumplidas, hacen con que la persona sufra sanción de parte del Estado o de otras personas de la sociedad; ya la ética dirige las acciones de cada miembro de la sociedad, para que haya una convivencia pacífica. Los principios de la Ética Social son de extrema importancia, pues nortean las acciones de las personas, así como el comportamiento de las organizaciones en la sociedad en que está inserida. Alonso, López y Castrucci (2006), describen seis principios clásicos de la Ética Social, ellos son: Dignidad de la Persona, Primacía del Trabajo, Primacía del Bien Común, Solidaridad, Subsidiariedad.

Ya, en el ámbito de las organizaciones, la Ética Empresarial no se refiere a la empresa en si apenas, sino a sus gestores y sus decisiones. Las acciones pueden ser juzgadas de acuerdo con los principios éticos, bajo reglas escogidas y usadas por los tomadores de decisión, estas acciones envuelven a los administradores, las normas sociales y corporativas, representadas por un Código de Ética propio. Un código de ética empresarial, para Alonso, López y Castrucci (2006), es un conjunto de normas éticas dictadas por la autoridad empresarial con vistas al bien común. El debe especificar 
las infracciones previsibles y la sanción correspondiente a cada una de ellas. Se presenta como un instrumento de realización de los principios, visión y misión de la empresa, sirve también, para orientar las acciones de los colaboradores y explicitar la postura social del negocio. Es criado por la empresa a partir de la realidad e de los problemas vividos, debe ser mencionado lo que cada empleado debe y no debe hacer de forma clara y también, especificar los comportamientos que una organización deberá asumir en su actuación en el mercado.

Bajo esta perspectiva, Ashley (2005), apunta la RS como todas las acciones que una empresa desarrolla y que puedan contribuir para la mejoría de la calidad de vida de la sociedad. Esas acciones comprenden y asumen las obligaciones de carácter moral, además de las establecidas en ley. En este abordaje, la RS puede ser evaluada sobre el punto de vista moral y ético.

\section{Dimensiones y Percepciones sobre la RSE}

Archie Carroll (1979), desarrolló uno de los modelos RSE más citados en las literaturas. Su teoría es representada por un modelo en forma de pirámide con cuatro responsabilidades conforme representación iconográfica (Figura I) a seguir:

Este modelo está fundamentado en cuatro tipos de responsabilidades (económica, legal, ética e discrecional) cuya empresa precisaría alcanzar para el desarrollo de sus negocios y así ser considerada socialmente responsable. La Responsabilidad Económica se localiza en la base de la pirámide, por ser el lucro, el objetivo principal de la existencia de las empresas. Además, tener responsabilidad económica es producir bienes y servicios que vengan a atender las necesidades de la sociedad con un precio justo que pueda garantir la continuidad de los negocios.

Ya la Responsabilidad Legal, se refiere a la visión que la sociedad tiene del comportamiento de la empresa, o sea, se es- pera que se obtenga los resultados deseados, obedeciendo la estructura legal y las exigencias legales, en que se refiere a las leyes municipales, del estado o federales.

La Responsabilidad Ética está relacionada con las actividades y acciones que la sociedad espera de la empresa, sin estar necesariamente fundamentada en la legalidad. Con base en la ética, los tomadores de decisión de las organizaciones deben actuar con equidad, justicia e imparcialidad, sin dejar de respectar los derechos individuales.

En fin, la Responsabilidad Discrecional de la empresa es guiada por la voluntad de ayudar a la sociedad contribuyendo con obras caritativas, instituciones de caridad, entre otros, sin ser impuesta por la economía, por la ley o por la ética, siendo esta, estrictamente opcional.A partir de estos fundamentos, surgirán otros debates y definiciones sobre la RSE que fueron mejorados a partir de la necesidad de buscar mayores diálogos con los stakeholders.

De acuerdo con el Instituto Ethos (20I I), la RSE es una forma de gestión que se define por la relación ética y transparente de la empresa con todos los públicos con los cuales ella se relaciona y por el establecimiento de metas empresariales compatibles con el desarrollo sustentable de la sociedad.

Ya, para la Unión Europea, el concepto RS seria aquello en que las empresas integran, de forma voluntaria, las preocupaciones sociales y ambientales en sus operaciones empresariales y en su interacción con las partes interesadas.

Del punto de vista normativo, específicamente por la regulación técnica de la ABNT/ISO 26000, denominada Social Accountability, se tiene, que las organizaciones socialmente responsables son aquellas que en sus acciones están dispuestas a asumir las responsabilidades por el impacto de sus actividades sobre la sociedad y el medio ambiente, en que esas acciones son consistentes con los intereses de la sociedad y con el desarrollo sustentable. Estas tienen comportamiento ético, cumplimento de las leyes aplicables y

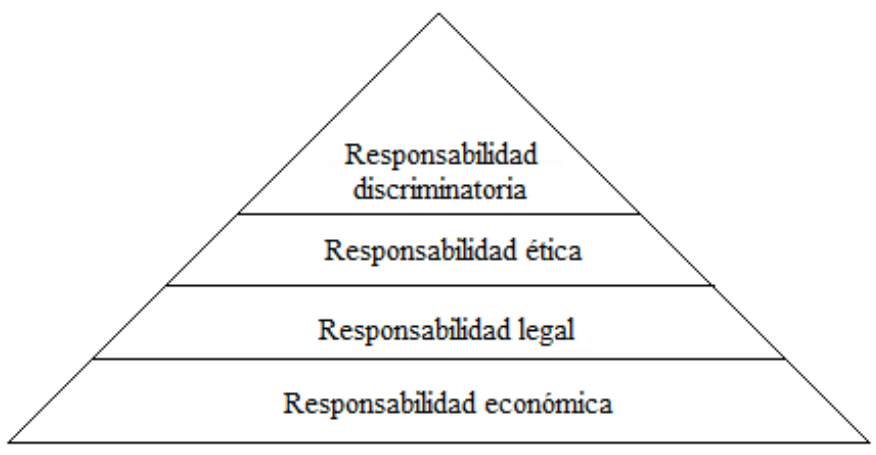

Figura I. Modelo de Responsabilidad Social.Adaptado de Carrol (1979).

ISSN: 07 I8-2724. (http://www.jotmi.org)

Journal of Technology Management \& Innovation (C) Universidad Alberto Hurtado, Facultad de Economía y Negocios. 
instrumentos intergubernamentales y estos principios se aplican en todas las actividades de la organización, o sea, en su cadena de negocios.

De esta forma, las organizaciones podrán ser evaluadas cuando el cumplimento de las normativas y la ISO 26000 auxiliaría los gestores de los emprendimientos en las cuestiones referentes a la RSE, mismo que con carácter voluntario visando la consciencia de las organizaciones para inserir en su propia cultura y en su misión.

Por el expuesto, se percebe que la RS es un tema ampliamente discutido, aunque organizaciones $y$ autores tengan formas distintas de la definir, su objetivo siempre será aumentar la calidad de vida de la sociedad y de las generaciones futuras, llevando a las empresas y todo el sistema en al cual está inserido, a utilizar los recursos disponibles conscientemente.

Por lo tanto, se entiende que, la construcción del concepto de la RSE está basada en la percepción de valores adquiridos por los sujetos en la sociedad y en la condición de consumidores de productos y servicios; estas percepciones, que afectan directamente en sus acciones como ser humano $y$ como profesionales de dichas organizaciones.

\section{Percepciones sobre la RSE y formación profesional}

Las instituciones de enseñanza técnica y superior en Brasil son responsables por la formación profesional de los trabajadores que actúan en las diversas organizaciones, además de la enseñanza básica curricular de los cursos, estas precisan de comprometerse con la formación ética y moral de éstos, de este modo formar profesionales preocupados con el bien estar de la sociedad.

A partir del año de 2005 la RS pasó a ser una de las dimensiones del instrumento de evaluación de la educación superior como parte de la política gubernamental de evaluación de la educación superior por intermedio del Sistema Nacional de Evaluación de la Educación Superior (SINAES), este hecho influenció la inserción del tema RSE en contexto de enseñar y aprender las acciones desarrolladas en alcance de las Instituciones de Enseñanza Superior (IES) en Brasil. El decreto de ley I086I/2004 confiere a las IES la responsabilidad por la inclusión social, desarrollo económico y social, defensa del medio ambiente, de la memoria cultural, producción artística y del patrimonio cultural.

En 2005, el sector educacional creó la Carta de Principios de la Educación Superior Brasileña Privada que determina, a través de la Asociación Brasileña de Mantenedoras de Educación Superior (ABMES), los valores de estas instituciones en cuanto negocios y el código de auto reglamentación es- tán siendo un marco para la inserción de la RS en IES, así, "la promoción de la enseñanza socialmente responsable abarcaría la formación de personas calificadas para inclusión en el mercado profesional e igualmente personas críticas y moralmente competentes, capaces de tomar decisiones frente la cuestiones éticas” (Bolan y Motta, 2007, p. 208).

Estos profesionales participan de las organizaciones y están involucrados directamente con los diversos niveles de responsabilidad dentro de las empresas, inclusive en aquellas con respecto a la RSE.

El perfil profesional cambió a lo largo de la historia del trabajo, actualmente tan importante cuanto tener competencias técnicas, es fundamental ser un profesional emprendedor, ético y socialmente responsable.

Así, las instituciones de educación públicas o privadas, formadoras de estos profesionales en nivel técnico, tecnológico y superior, deben engancharse en la formación en sintonía con las creencias y valores, misión y visión de las empresas en las cuales van actuar, pero también, conscientes de las consecuencias que su acción profesional es capaz de provocar impactos positivos o negativos junto a los stakeholders. Los estudios desarrollados por Barnett (2007, p. 796), sobre la influencia de los stakeholders sobre la capacidad y la variabilidad de los retornos financieros apuntaran que las empresas que "desarrollan relacionamiento de confianza mutua y cooperación con stakeholders obtienen una ventaja competitiva sobre aquellas que no".

Por eso, es posible comprender que exista una presión de la sociedad para que las organizaciones adopten prácticas socialmente responsables. Conforme Aguilera et. al. (2007), las empresas que no respondieren a esa demanda perderán la legitimidad juntos a sus stakeholders, de este modo, es posible comprender porque las empresas están adoptando practicas de RS, bien cómo es posible verificar la existencia de esa postura por las universidades (Atakan y Eker, 2007). En ese contexto, cuando las universidades forman profesionales con la percepción socialmente responsable, contribuyen para que las empresas adopten prácticas socialmente responsables.

Para Muijen (2004), las universidades deben tener como deber educacional enseñar los principios de la RS para con los alumnos de graduación. Las IES, por su naturaleza, tienen un papel fundamental en el desarrollo de un país, su misión es transmitir y producir nuevos conocimientos por intermedio de las actividades de enseñanza, investigación y extensión, de manera que estos conocimientos puedan intervenir de forma positiva en la calidad de vida de la sociedad.

En Brasil, las universidades ya tienen dado pasos largos en

ISSN: 07 I8-2724. (http://www.jotmi.org)

Journal of Technology Management \& Innovation (c) Universidad Alberto Hurtado, Facultad de Economía y Negocios. 
sentido de discutir la profesionalización de este sector. Denominados tecnología social, visa buscar la proposición de estrategias de inclusión social a través del sector privado con alternativas creativas y emprendedoras, premios, eventos, campañas publicitarias y partidarios entre el público y el privado. Sin embargo, todavía encuentran dificultades para interiorizar estos asuntos, de modo estratégico, en proyectos políticos pedagógicos, contenidos disciplinares y interdisciplinarios y que por veces parte del cuerpo docente desconoce la profundización incipiente sobre los temas de la RSE.

Muchos debates ya han sido desarrollados, basados de las universidades sobre la RSE, una vez que las IES son las formadoras de opinión que deben tener el papel de sensibilizar y provocar los debates acerca de la RSE y humana.

\section{Metodología}

Para atingir el objetivo de estudio, fue desarrollada una investigación exploratoria, cuyos resultados permitirían identificar la percepción de los alumnos de graduación de la UTFPR sobre el asunto tratado en este articulo.

\section{Muestra}

Los alumnos que participaran de la investigación fueron seleccionados por el criterio de accesibilidad. La UTFPR campus de Ponta Grossa, cuenta con 2.153 alumnos matriculados en los cursos de ingeniería, tecnología y técnico.

La muestra del estudio se constituyó de 212 alumnos, siendo 58 alumnos de cursos de ingeniería, 120 alumnos de cursos de tecnología y 34 alumnos de cursos técnicos, representando una muestra de aproximadamente $10 \%$.

\section{Instrumento de pesquisa}

Como instrumento de colecta de datos, fue elaborado un cuestionario basado en los Indicadores de RSE del Instituto Ethos (2003), conteniendo $2 \mathrm{I}$ cuestiones. Las dos primeras cuestiones fueron para identificar la faja etaria de los encuestados, como el curso en que estaba matriculado. De las 19 cuestiones siguientes, 17 fueron elaboradas en escala de tipo Escala de Likert de tres puntos (desacuerdo, no sé decir, acuerdo) siendo que la cuestión de número 7 rec- ibió un tratamiento diferenciado en su terminología (bajo, medio y alto) debido la cuestión de concordancia con la cuestión investigada. Sin embargo, es correspondiente con las demás cuestiones. Las dos últimas cuestiones fueron de selección múltiple en que los alumnos podrían marcar más de una alternativa como respuesta para mejor representar la cuestión investigada.

Este cuestionario fue estructurado en tres dimensiones básicas. Siendo que en el primero, se buscó identificar la percepción de los alumnos referente a la RS en las empresas. Para responder la segunda dimensión, fue investigada la percepción de los alumnos en cuanto a la relación de la RS en la universidad y finalmente, para la tercera dimensión, fueron identificadas la percepción de la RS que los alumnos tienen de sí mismos.

La Tabla I presenta detenidamente las cuestiones referentes a cada dimensión investigada para ese artículo.

Así, las cuestiones referentes a la primera dimensión tiene por objetivo identificar la percepción de los alumnos sobre la RS de las organizaciones, la segunda dimensión visa identificar la percepción de los alumnos sobre la RS en la UTFPR y las cuestiones referentes a la tercera dimensión son para verificar cual percepción que los alumnos tienen sobre la RS de sí mismos.

El cuestionario fue elaborado en la herramienta del Google docs y enviado vía web, a los 2.153 alumnos juntamente con un texto explicativo sobre el objetivo de la investigación y las instrucciones para rellenar, sin embargo, después de 90 días de la fecha de envío, de los cuestionarios, apenas 215 cuestionarios retornaron rellenados a los investigadores.

\section{Tratamiento de los datos}

Fue identificado el grado de concordancia o discordancia de cada una de las cuestiones, obteniéndose por medio de estadística simple, el porcentual de las respuestas para cada punto de la escala en relación al número total de respuestas. Con excepción de las cuestiones I, 2, 20 y 2 I que no utilizaron esta escala. Para las dos últimas cuestiones, la investigación elaboró en forma de gráfico, la frecuencia con que los estudiantes rellenaron las cuestiones, recordando que no podrían ser rellenadas más de una opción.

\begin{tabular}{|l|l|l|l|}
\hline Percepción de los alumnos cuanto a la Responsabilidad Social & & & \\
\hline & Empresas & UTFPR & Si mismos \\
\hline Cuestiones & $3,4,5,6,7,8,9,10,11,12,13$ & $14,15,17,18$ & $16,19,20,21$ \\
\hline
\end{tabular}

Tabla I. Percepción de los Alumnos cuanto la Responsabilidad Social. Los autores (20I I).

ISSN: 07 I8-2724. (http://www.jotmi.org)

Journal of Technology Management \& Innovation (c) Universidad Alberto Hurtado, Facultad de Economía y Negocios. 


\section{Presentación y Análisis de los Resultados}

\section{Edad}

La faja etaria de los encuestados son presentadas en el Gráfico I.

Se percibe claramente que la mayoría de los alumnos se encuentran en la faja etaria de 19 a 21 años, más precisamente, fueron 82 encuestados, de los demás alumnos, 56 están con edad entre 15 a 18 anos, 34 están en la faja etaria de 22 a 24 años, 21 se encuentran entre 25 a 29 anos, 12 alumnos tienen entre 30 a 34 años y solamente 7 encuestados están arriba de los 35 años.

\section{Área del curso}

El Gráfico 2 presenta el porcentual de los alumnos cursando las áreas de ingeniería, tecnología y técnico que participaron de la investigación.

De los encuestados, 58 están matriculados en el área de ingeniería, 34 están cursando el técnico y 120 alumnos son de los cursos de tecnología.

\section{Percepción de los alumnos sobre la RSE}

Cuanto a los resultados obtenidos referentes a la percepción de los alumnos acerca del tema RS en relación a las tres dimensiones investigadas, la Tabla 2 presenta los porcentuales para cada cuestión respondida.

Los resultados revelan que los alumnos tienen un buen entendimiento cuanto a la diferencia de concepto entre la RS y Filantropía. Hecho que va al encuentro de la observación de Oliveira (2008), al afirmar que no se puede confundir el concepto de la RS con el concepto de filantropía, mismo que el porcentual acentuado de $8 \mathrm{I}, 3 \%$ de los encuestados tenga

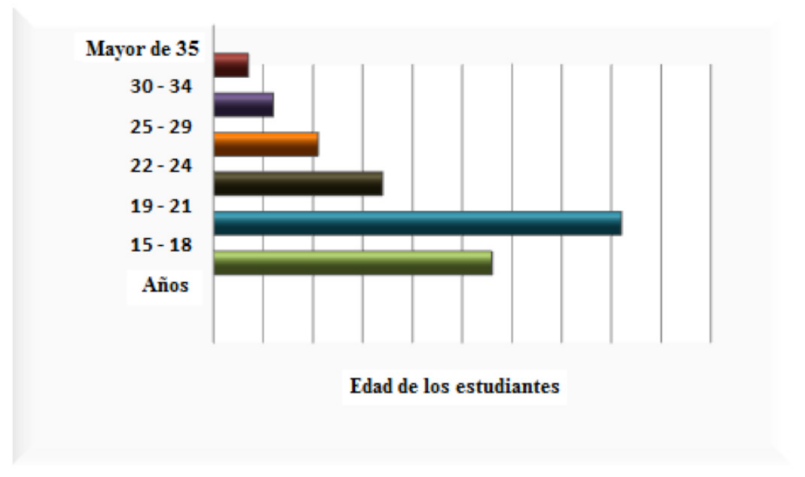

Gráfico I. Edad de los encuestados. Datos de la investigación (20II). concordado sobre la existencia de un compartimiento entre empresas y gobiernos en la promoción social y en desarrollo de la nación, 96,2\% de los alumnos acreditan que las empresas precisan se responsabilizar por sus acciones que generan problemas sociales. Para eso, algunas empresas divulgan en general sus proyectos sociales en los cuales los empleados y toda la comunidad pueden participar. En ese caso, de acuerdo con Alonso, López y Castrucci (2006), retrata el Brasil de los años 80 en forma de acciones filantrópicas consideradas como inversión social al practicar acción social sobrepuesta a la gestión tradicional. Ese dato demuestra que, a pesar de Bower (1954) ter identificado, hace mucho tiempos, las obligaciones de los lideres, cuanto a sus decisiones y adopción de políticas, la responsabilidad de los agentes empresariales cuanto al respecto de los valores y objetivos sociales todavía se cargará por la sociedad en los días actuales. Sin embargo, la acusación al respecto de la responsabilidad se extiende a las acciones de otras empresas.

Aproximadamente $86 \%$ de los alumnos discordan en cuanto a la alternativa que afirma no haber necesidad de las empresas contribuir con acciones que beneficien a la sociedad, aun cuando la empresa no es la causa del problema, a pesar de obtener un beneficio o no de esas acciones.

Cuanto a la importancia de la RS para el desarrollo sostenible de la sociedad, el $73 \%$ de los alumnos cree que el grado de actividad de este factor es alto. Esta percepción está alineada a la visión económica de Alonso (2006), pero está en línea con la exigencia de los clientes como para requerir acciones de instituciones socialmente responsables.

Para la creación de una imagen positiva, el $93 \%$ de los encuestados están de acuerdo que las acciones de responsabilidad social contribuyen para eso, sin embargo, estas acciones se invierten en forma de beneficios para las empresas, como tal, debe ser una inversión a largo plazo.

Los alumnos se dan cuenta de que la RS es un diferencial y que aumenta la competitividad empresarial, sin embargo, los resultados muestran casi la misma proporción, que los

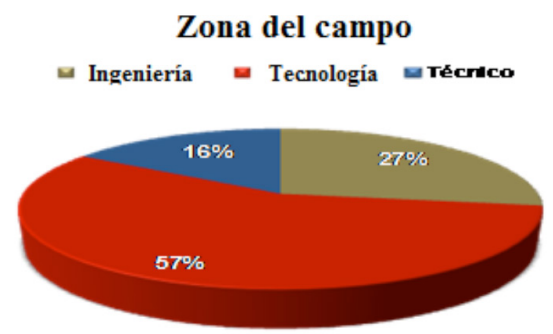

Gráfico 2. Área del Curso. Datos de la investigación (201 I).

ISSN: 07I 8-2724. (http://www.jotmi.org)

Journal of Technology Management \& Innovation (c) Universidad Alberto Hurtado, Facultad de Economía y Negocios. 
alumnos no creen o no conocen las acciones socialmente responsables de las empresas en Brasil, pero incluso sin creer y sin saber que estas acciones no están de acuerdo, el $81,3 \%$ dicen que es una moda pasajera. Estos datos revelan opiniones de los alumnos sobre el comportamiento de la empresa, que se extiende entre la Responsabilidad Legal y Ética en la pirámide de Carrol (1979).

La percepción de los alumnos sobre la RS de la Universidad Cuanto a la percepción de los alumnos para identificar las acciones de responsabilidad Social por la UTFPR, la Tabla 3 presenta los resultados.

Para la segunda dimensión, propuesta en este trabajo, $74,5 \%$ de los alumnos concuerdan que la UTFPR contribuye a la formación de posicionamiento responsable. También hay un grado significativo de concordancia, $70,2 \%$ en cuanto la institución sea consciente de su Responsabilidad Social. Situando la UTFPR en el topo de la pirámide del modelo de Carrol (1979), Responsabilidad Diccionarista, cuanto a la adopción de las acciones que auxilian la sociedad sin ser impuesta por la economía, por la ley o por la ética, motivada por una opción consciente de la importancia de las políticas que conducen la institución e del impacto de las acciones en la formación profesional de los alumnos.

Así, la UTFPR cumple, de acuerdo con Muijen (2004), con el deber educacional de enseñar los principios de la RS a los alumnos, atendiendo la misión de discutir, producir y transmitir conocimientos nuevos por medio de la tecnología social, mismo que encuentre cierta dificultad en integrar sus proyectos políticos pedagógicos con los contenidos interdisciplinares y con el cuerpo docente de la institución.

\section{Percepción de los Universitarios}

Para la percepción de los alumnos sobre la comprensión propia de la Responsabilidad Social, la Tabla 4 presenta los resultados.

Los resultados de la tercera dimensión de la investigación, principalmente en las cuestiones de selección múltiple,

\begin{tabular}{|l|l|l|l|}
\hline $\begin{array}{l}\text { Percepción de los alumnos cuanto la Responsabilidad Social de las } \\
\text { Empresas (\%) }\end{array}$ & & \\
\hline Cuestiones & Desacuerdo & No sé decir & Concuerdo \\
\hline 3. RS y Filantropía son sinónimos. & 59,6 & 8,7 & 31,7 \\
\hline $\begin{array}{l}\text { 4.Al envolverse en actividades socialmente responsables, las empresas } \\
\text { comparten con el gobierno el papel de promover el bien estar y el } \\
\text { desarrollo social. }\end{array}$ & 13,0 & 5,8 & 81,3 \\
\hline $\begin{array}{l}\text { 5. Las empresas deben se responsabilizar por los problemas sociales } \\
\text { creados por sus acciones. }\end{array}$ & 1,9 & 1,9 & 96,2 \\
\hline $\begin{array}{l}\text { 6. Las empresas no precisan intentar resolver problemas sociales } \\
\text { creados por otras empresas, una vez que estas acciones retornan en } \\
\text { forma de lucro. }\end{array}$ & 85,6 & 9,6 & 4,8 \\
\hline $\begin{array}{l}\text { 7. La importancia de la RS para el desarrollo sustentable de la sociedad } \\
\text { pode ser considerada de grado. }\end{array}$ & Bajo & Medio & Alto \\
\hline & 3,8 & 23,1 & 73,1 \\
\hline $\begin{array}{l}\text { 8. Programas de acciones sociales (o RS) contribuyen en la construc- } \\
\text { ción de una imagen positiva para la empresa. }\end{array}$ & 1,4 & 5,3 & 93,3 \\
\hline $\begin{array}{l}\text { 9. Contribuir con la solución de los problemas sociales pode ser lucra- } \\
\text { tivo para las empresas. }\end{array}$ & 3,8 & 16,8 & 79,3 \\
\hline I0. Responsabilidad Social es una inversión de largo plazo. & 10,6 & 13,0 & 76,4 \\
\hline $\begin{array}{l}\text { II. Responsabilidad Social es un elemento importante para el aumento } \\
\text { de la competitividad empresarial. }\end{array}$ & 13,9 & 22,1 & 63,9 \\
\hline $\begin{array}{l}\text { I2. Actualmente existen muchas empresas socialmente responsables } \\
\text { en Brasil. }\end{array}$ & 46,6 & 49,5 & 3,8 \\
\hline I3. El tema sobre la Responsabilidad Social es pasajero. & 81,3 & 13,9 & 4,8 \\
\hline
\end{tabular}

Tabla 2. Percepción de los Alumnos cuanto a la RSE. Datos de la investigación (20I I).

* Tratamiento diferenciado en la terminología.

ISSN: 07 I8-2724. (http://www.jotmi.org)

Journal of Technology Management \& Innovation (c) Universidad Alberto Hurtado, Facultad de Economía y Negocios. 


\begin{tabular}{|l|l|l|l|}
\hline La percepción de los alumnos cuanto la Responsabilidad Social de la UTFPR (\%) & & & \\
\hline Cuestiones & Discuerdo & No sé decir & Concuerdo \\
\hline I4. La UTFPR es una institución consciente de su RS & 5,3 & 24,5 & 70,2 \\
\hline I5. La UTFPR ejerce proyecto e/o acción de Responsabilidad Social & 4,3 & 29,3 & 66,3 \\
\hline $\begin{array}{l}\text { I7. La UTFPR contribuye para la formación de posicionamiento socialmente } \\
\text { responsable en sus alumnos }\end{array}$ & $\mathrm{II,5}$ & 13,9 & 74,5 \\
\hline $\begin{array}{l}\text { I8. Los conceptos de la RS son definidos/presentados/discutidos en disciplinas } \\
\text { del curso al cual frecuento }\end{array}$ & $34, \mathrm{I}$ & $\mathrm{I4,9}$ & $5 \mathrm{I,0}$ \\
\hline
\end{tabular}

Tabla 3. Percepción de los Alumnos cuanto la RS de la UTFPR. Datos de la investigación (20I I).

\begin{tabular}{|c|c|c|c|}
\hline $\begin{array}{l}\text { Percepción de los alumnos cuanto la Responsabilidad } \\
\text { Social de si mismo (\%) }\end{array}$ & & & \\
\hline Cuestiones & Discuerdo & No sé decir & Concuerdo \\
\hline $\begin{array}{l}\text { 16. Las acciones de RS divulgadas por las empresas influ- } \\
\text { encian en la decisión de compra de los consumidores }\end{array}$ & 8,7 & 19,7 & 71,6 \\
\hline 19. Puedo ser considerado un consumidor consciente & 17,3 & 18,8 & 63,9 \\
\hline Cuestiones de selección Múltiple & Alternativas & (\%) & \\
\hline \multirow{5}{*}{$\begin{array}{l}\text { 20.Al comprar algún producto o servicio, generalmente } \\
\text { opto por: }\end{array}$} & Más económicos & 64,9 & \\
\hline & Con producción más limpia & 32,2 & \\
\hline & Reciclables & 35,1 & \\
\hline & $\begin{array}{l}\text { Producción de empresas que se } \\
\text { dicen socialmente responsables }\end{array}$ & 29,3 & \\
\hline & Calidad & 83,2 & \\
\hline \multirow{10}{*}{$\begin{array}{l}21 . \text { De acuerdo con su percepción, cuales alternativas } \\
\text { representan las mejores prácticas de las empresas en } \\
\text { relación a la RS }\end{array}$} & Donaciones monetarias & 10,1 & \\
\hline & $\begin{array}{l}\text { Respecto a los derechos hu- } \\
\text { manos }\end{array}$ & 82,2 & \\
\hline & Preservación del medio ambiente & 93,8 & \\
\hline & $\begin{array}{l}\text { Reducción del consumo de agua } \\
\text { y energía }\end{array}$ & 76,0 & \\
\hline & Reciclaje & 83,7 & \\
\hline & $\begin{array}{l}\text { Prohibir el uso de técnicas } \\
\text { comerciales anti éticas }\end{array}$ & 44,2 & \\
\hline & Instalarse en comunidades pobres & 9,6 & \\
\hline & Combate a la corrupción & 45,2 & \\
\hline & $\begin{array}{l}\text { Comprometimiento con practi- } \\
\text { cas a favor del trabajo }\end{array}$ & 52,9 & \\
\hline & Otros & 7,7 & \\
\hline
\end{tabular}

Tabla 4. Percepción de los Alumnos en cuanto a la RS de sí Mismos. Datos de la investigación (20II).

ISSN: 07 I8-2724. (http://www.jotmi.org)

Journal of Technology Management \& Innovation (C) Universidad Alberto Hurtado, Facultad de Economía y Negocios. 
muestran una mezcla de las cuatro responsabilidades de Carrol (1979). Los alumnos poseen una visión más volteada a las cuestiones económicas de la RS, esto es, $64,9 \%$, acreditan que la producción de bienes y servicios para suplir las necesidades de la sociedad precisa cobrar apenas el precio que proporcione la continuidad de los negocios cuando optan por productos más económicos. Así, hay coherencia en la observación de Barnett (2007) sobre la influencia de los stakeholders en los retornos financieros de las empresas al desarrollar la confianza y la cooperación proporcionando las empresas ventaja competitiva sobre sus competidores.

Por consiguiente, las cuestiones de calidad $83,2 \%$, respecto de los derechos humanos $82,2 \%$, preservación del medio ambiente $93,8 \%$, reducción del consumo de agua $76 \%$ y reciclaje $83,7 \%$ demuestran la Responsabilidad Legal y Responsabilidad Ética adecuándose al concepto de la Unión Europea donde las empresas integran preocupaciones sociales y ambientales en las actividades y en las relaciones con sus stakeholders (partes interesadas), corroborando con Aguilera et. al. (2007), cuanto la legitimidad de las prácticas de la RS de las empresas contribuyendo para las prácticas socialmente responsables.

\section{Consideraciones finales}

A pesar de la intensificación de los debates sobre el tema, aún existe una visión equivocada cuando se confunde la RSE como apenas un cumplimento de las obligaciones sociales, filantropía (donaciones) o acciones sociales. La investigación permitió reflexionar sobre el abordaje de la RSE y las percepciones de los alumnos de la Universidad Tecnológica Federal en este asunto.

En la primera dimensión, percepción de los alumnos referente a la Responsabilidad Social en las empresas, ha sido constatado que la responsabilidad y la resolución de los problemas sociales inherentes a la actuación de los procesos productivos de las empresas precisan ser asumidos por las mismas una vez que causan impacto en la imagen y el desarrollo sustentable de la empresa.

Para la segunda dimensión, sobre la percepción con relación a la universidad, se percibe que la institución de enseñanza en la cual la investigación ha sido realizada asume el papel de integrar las políticas pedagógicas a los contenidos de las disciplinas al mismo tiempo en que se debe crear nuevos conocimientos. Bajo la óptica de los alumnos hay el entendimiento de las acciones compartidas con las acciones gubernamentales para la promoción del bien estar, del desarrollo social, de la percepción de los proyectos y acciones realizadas por la UTFPR que contribuye para el posicionamiento responsable en la formación profesional de los alumnos.
Finalmente, en cuanto a la tercera dimensión, la percepción que los alumnos tienen sobre la Responsabilidad Social de sí mismos sobre la RS, los datos demuestran que la mayoría de los alumnos acreditan ser consumidores conscientes por priorizar calidad en sus compras y que las mejores prácticas con relación a la Responsabilidad Social son aquellas que preservan el medio ambiente, que practican la reciclaje $y$ que respectan los derechos humanos.

Así, los investigadores entienden que la visión adecuada por parte de la sociedad, estudiantes y futuros profesionales sobre la RSE será posible solamente si hubiera el intercambio de la informaciones, integración del tema en la formación, tenga reflexiones y debates sobre el tema de forma que pueda crear una nueva cultura en la cual la concientización y las acciones sean integradas en el día a día de los ciudadanos. 


\section{Agradecimientos}

Los autores agradecen el apoyo de La Coordenação de Aperfeiçoamento de Pessoal de Nível Superior (CAPES) y Programa de Assistência ao Ensino (PAE) para la realización de este trabajo.

\section{Referencias}

ABNT. NBR 26000:20I0. Diretrizes sobre responsabilidade social. Rio de Janeiro, 2010.

ATAKAN, M. G. S., Eker,T. (2007). Corporate identity of a socially responsible university - a case from the Turkish higher education sector. Journal of Business Ethics, 76, 55-68.

ASHLEY, P. A. (2005). Ética e responsabilidade social nos negócios. São Paulo: Saraiva.

AGUILERA, R.V., Rupp, D. E., Williams, C. A., Jyoti, G. (2007). Putting the $S$ Back in corporate social responsibility: a multilevel theory of social change in organizations. Academy of Management Review, 32(3), 836-863.

ABMES. ASSOCIAÇÃO BRASILEIRA DE MANTENEDORAS DE ENSINO SUPERIOR. (20I3). Código de auto-regulamentação das instituições e ensino superior. http://www. abmes.org.br/abmes/ [Accessed March 08, 2013].

ALONSO, R. F., López, F. G., Castrucci, P. L. (2006). Curso de ética em administração. São Paulo:Atlas.

BARNETT, M. L. Stakeholder influence capacity and the variability of financial returns to corporate social responsibility. Academy of Management Review, 32(3), 794-816.

BRASIL. (20I3). Ministério da Educação. Lei $10.86 \mid$, de 14 de abril 2004 institui o Sistema Nacional de Avaliação da Educação Superior-SINAES e dá outras providências. http:// portal.inep.gov.br/superior-condicoesdeensino-legislacao_ normas [Accessed March 10, 20I3].

BOLAN, V., Motta, M. V. (2007). Responsabilidade social no ensino superior. Revista de Educação, I0(I0), 204-2I0.

BOWER, H. R. (1953). Social Responsibilities of the businessman. New York: Haper \& Row.

CARROL,A. B. (I99|).The Pyramid of Corporate Social Responsibility: Toward the Moral Management of Corporate Stakeholders. Business Horizons, 34(4), 39-48.
CARROL, A. B. (1979). A three-dimensional conceptual model of corporate performance. Academy of Management Review, 4(4), 497-505.

INSTITUTO ETHOS DE EMPRESAS E RESPONSABILIDADE SOCIAL. (20II). O que é Responsabilidade Social. http://wwwl .ethos.org.br/EthosWeb/pt/29/o_que_e_ rse/o_que_e_rse.aspx [Accessed May 20, 20I I].

ETHOS, SEBRAE. (2003). Responsabilidade Social Empresarial para Micro e Pequenas Empresas: passo a passo. São Paulo.

GRAYSON, D., Hodges, A. (2002). Compromisso social e gestão empresarial. São Paulo: Publifolha.

FRIEDMAN, M. (1979). The Social Responsability of Business is to Increase its Profits. The New York Times Magazine, I3, $\mathrm{I}-12$.

OLIVEIRA,J.A.P. (2008). Empresas na sociedade: sustentabilidade e responsabilidade social. Rio de Janeiro: Elsevier.

MUIJEN, H. S. C. A. (2004). Corporate social responsibility starts at university. Journal of Business Ethics, 53(I), 235-246.

TACHIZAWA, T., Andrade, R. O. B. (2008). Gestão Socioambiental: Estratégias na nova era da sustentabilidade. Rio de Janeiro: Elsevier.

TENÓRIO, F. G. (org.). (2006). Responsabilidade social empresarial: teoria e prática. 2 ed. Rio de Janeiro: Editora FGV. 